\title{
Aspectos dietéticos das refeições oferecidas por empresas participantes do Programa de Alimentação do Trabalhador na Cidade de São Paulo, Brasil
}

\author{
Ana Paula Gines Geraldo, ${ }^{1}$ Daniel Henrique Bandoni ${ }^{1}$ \\ e Patrícia Constante Jaime ${ }^{1}$
}

Como citar

Geraldo APG, Bandoni DH, Jaime PC. Aspectos dietéticos das refeiç̃os oferecidas por empresas participantes do Programa de Alimentação do Trabalhador na Cidade de São Paulo, Brasil. Rev Panam Salud Publica. 2008;23(1):19-25.

RESUMO Objetivo. Avaliar os aspectos dietéticos das refeições oferecidas por empresas inscritas no Programa de Alimentação do Trabalhador (PAT) na Cidade de São Paulo, Brasil, em relação às recomendações nutricionais do Guia Alimentar para a População Brasileira do Ministério da Saúde. Métodos. Foram investigadas 72 empresas, caracterizadas conforme setor (indústria, serviços ou comércio), porte (micro, pequenas, médias ou grandes), modalidade do PAT (autogestão, gestão terceirizada do tipo refeição transportada ou gestão terceirizada do tipo preparo e distribuição de refeição na empresa) e supervisão de nutricionista (sim ou não). A quantidade per capita dos alimentos foi determinada nos cardápios de 3 dias de almoço, jantar e ceia. $O$ valor nutricional das refeições foi definido com base nas variáveis energia, carboidrato, proteina, gorduras totais, gordura poliinsaturada, gordura saturada, gordura trans, açúcares livres, colesterol e frutas e hortaliças.

Resultados. A maioria dos cardápios teve baixa oferta de frutas e hortaliças $(63,9 \%)$ e gordura poliinsaturada $(83,3 \%)$ e excesso de gorduras totais $(47,2 \%)$ e colesterol $(62,5 \%)$. O agrupamento 2, composto principalmente por empresas de médio e grande porte do setor industrial e de serviços, com gestão terceirizada e supervisão de nutricionista, teve, em média, refeições com maior valor energético $(P<0,001)$, maior porcentagem de gorduras poliinsaturadas $(P<0,001)$, maior quantidade de colesterol $(P=0,015)$ e maior quantidade de frutas $e$ hortaliças $(P<0,001)$ do que o agrupamento 1 , composto por micro e pequenas empresas do setor de comércio, tendo autogestão como modalidade e sem supervisão de nutricionista. Quanto à adequação dos cardápios oferecidos às metas do Guia Alimentar para a População Brasileira, as refeições do agrupamento 2 foram mais adequadas em relação à oferta de frutas e hortaliças $(P<0,001)$. Em contrapartida, o agrupamento 1 apresentou maior adequação da quantidade de colesterol $(P=0,05)$.

Conclusões. É necessária uma ação mais direcionada aos gestores de empresas e aos responsáveis pelas unidades de alimentação e nutrição, principalmente no grupo de empresas que não tem a supervisão de nutricionista.

Palavras-chave Programas e políticas de nutrição e alimentação, promoção da saúde, doença crônica, planejamento de cardápio, local de trabalho, Brasil.

Universidade de São Paulo, Faculdade de Saúde Pública, Departamento de Nutrição, São Paulo (SP), Brasil. Enviar correspondência para Patrícia
Avenida Dr. Arnaldo 715, CEP 01246-904, São Paulo, SP, Brasil. E-mail: constant@usp.br 
Atualmente, são muitas as evidências científicas que comprovam a relação entre a alimentação e as doenças crônicas em adultos (1-4). Com base nesses estudos, e considerando o aumento na prevalência das doenças crônicas não transmissíveis e da obesidade em todo o mundo, a Organização Mundial da Saúde (OMS) propôs, em 2004, uma estratégia global de prevenção dessas doenças, apoiada na promoção de padrões saudáveis de alimentação e de estilos de vida ativos (5). Essa estratégia considera o local de trabalho como responsável por estimular as escolhas saudáveis de alimentação e a prática de atividade física entre os trabalhadores. Como mostra a literatura, os locais de trabalho são um excelente espaço para oferecer programas de promoção da saúde, em particular aqueles voltados para a redução de fatores de risco para doenças cardiovasculares $(6,7)$.

Em 2005, o Ministério da Saúde do Brasil lançou o Guia Alimentar para a População Brasileira (8), cujo objetivo é a implementação das recomendações preconizadas pela OMS (5). Além disso, o Brasil possui, desde 1977, um programa de alimentação voltado à população trabalhadora. O Programa de Alimentação do Trabalhador (PAT) tem como objetivo principal a melhoria das condições nutricionais dos trabalhadores, com repercussões positivas para a qualidade de vida, a redução de acidentes de trabalho e o aumento da produtividade (9). A partir de 2002, incorporou-se a promoção da saúde como eixo central do PAT (10).

Contudo, alguns estudos (11-13) têm demonstrado que o programa não vem atingindo o seu objetivo de promoção da saúde por meio da oferta de alimentação saudável, evidenciando uma oferta excessiva de lipídios e energia e elevada prevalência de obesidade e dislipidemia entre os trabalhadores beneficiados pelo PAT. Para que o PAT possa atingir o seu objetivo e se consolidar como um programa de promoção da saúde e alimentação saudável no ambiente de trabalho, é preciso que a alimentação oferecida aos beneficiados seja adequada às mais recentes recomendações nutricionais e ao novo cenário epidemiológico brasileiro, caracterizado pelo aumento da obesidade, do diabetes, das doenças cardíacas e de certos tipos de câncer. Nesse contexto, poucos estudos examinaram o PAT a partir da perspectiva da promoção da alimentação saudável no local de trabalho. Assim, o presente estudo foi desenvolvido com o objetivo de avaliar os aspectos dietéticos das refeições oferecidas por empresas inscritas no PAT em relação às recomendações nutricionais do Guia Alimentar para a População Brasileira.

\section{MATERIAIS E MÉTODOS}

O presente estudo transversal envolveu uma amostra aleatória de 93 empresas localizadas na Cidade de São Paulo e inscritas no PAT, tendo como critério de inclusão oferecer refeições diretamente no local de trabalho (empresas cadastradas nas modalidades autogestão, gestão terceirizada, preparo e distribuição de refeição e refeição transportada). Foram descartadas as empresas que oferecem benefícios como vale ou tíquete-refeição, cesta básica e outros.

São Paulo, a capital do Estado de São Paulo, é a maior cidade do Brasil. Segundo os dados do Instituto Brasileiro de Geografia e Estatística (IBGE) (14), sua população é superior a 11 milhões de habitantes. Com o maior produto interno bruto (PIB) do País, São Paulo concentra importantes corporações globais dos diversos setores da indústria, comércio, serviços e finanças. A participação do Estado de São Paulo no PAT é de $36,69 \%$, o que equivale a aproximadamente $51 \mathrm{mil}$ empresas. Estima-se que a participação da Cidade de São Paulo no PAT seja de 60 a $70 \%$ desse total.

O banco de dados das empresas inscritas no PAT na Cidade de São Paulo, utilizado no sorteio da amostra, foi fornecido pela coordenação geral do PAT no Ministério do Trabalho e Emprego. Esse banco continha informações de todas as empresas cadastradas, independentemente da modalidade de gestão. A partir daí, foram selecionadas aquelas que atendiam o critério de inclusão para posterior sorteio da amostra. Dessa forma, o tamanho inicial da amostra, de 93 empresas, correspondeu a $12,9 \%$ das empresas cadastradas no PAT no ano de 2003 nas modalidades de interesse.

A coleta de dados ocorreu entre outubro de 2003 e março de 2004. Ao final dessa etapa houve 21 perdas (22\%) (11 recusas e 10 empresas não encontradas). Assim, foram investigadas $72 \mathrm{em}-$ presas, o que corresponde a $9,98 \%$ das empresas cadastradas no ano de 2003 nas modalidades de interesse.

\section{Avaliação da adequação da refeição}

Os cardápios de 3 dias das grandes refeições (almoço, jantar e ceia) foram avaliados a partir das quantidades per capita dos alimentos e preparações fornecidos pelas empresas aos trabalhadores. Optou-se por avaliar cardápios de 3 dias, e não de apenas 1 dia, para controlar o possível erro aleatório da medida que poderia ocorrer se o dia selecionado não fosse característico do padrão de planejamento do cardápio.

As quantidades per capita oferecidas nas refeições foram calculadas com base no receituário padrão da unidade de alimentação e nutrição de cada empresa. Para as unidades que não possuíam o receituário, calculou-se a quantidade per capita dividindo-se a quantidade de alimento utilizada, obtida através da saída de estoque, pelo número total de refeições servidas no dia. As quantidades per capita também foram corrigidas pelo indicador de partes não comestíveis determinadas no Estudo Nacional de Despesas Familiares (ENDEF) de 1974 e 1975 (15).

Os cardápios foram analisados com o software Virtual Nutri, que teve a sua base de dados atualizada inicialmente com dados da Tabela de Composição de Alimentos (TACO) (16) e adicionalmente com dados da tabela de composição do Ministério de Agricultura dos Estados Unidos versões 16 e 17 (17). Preferencialmente, foram utilizados os dados da TACO; porém, quando o alimento não estava listado nessa tabela, foram utilizados os dados norteamericanos. Foi considerada a média 
aritmética dos 3 dias para a determinação das quantidades de cada nutriente oferecido pelas empresas.

O valor nutricional das refeições foi definido com base nas variáveis: energia, carboidrato, proteína, gorduras totais, gordura poliinsatorada, gordura saturada, gordura trans, açúcares livres, colesterol e frutas e hortaliças. Para avaliar a adequação dos cardápios, foram utilizadas as metas de ingestão de nutrientes recomendadas pelo Guia Alimentar para a População Brasileira (8) (tabela 1), que estão de acordo com as recomendações da OMS (4).

De acordo com a portaria que alterou os parâmetros nutricionais do PAT (18), as refeições principais devem fornecer de 30 a $40 \%$ do valor energético total diário, tendo como base uma dieta de $2000 \mathrm{kcal}$. Para estabelecer a oferta de colesterol adequada, considerou-se a recomendação preconizada pelo Ministério da Saúde (8), de até $300 \mathrm{mg}$ /dia. Assim, o valor correspondente a $40 \%$ da oferta diária equivale a $120 \mathrm{mg}$ de colesterol. A mesma portaria (18) também preconiza que os cardápios devem oferecer pelo menos uma porção de frutas e uma de legumes ou verduras nas refeições principais. Assim, a recomendação do Ministério da Saúde (8) de oferecer no mínimo 5 porções ao dia de frutas e legumes - $\mathrm{O}$ equivalente a $400 \mathrm{~g} / \mathrm{dia}$ - foi adaptada para duas porções de frutas e legumes, ou 160 g, por refeição, conforme preconizado pela Portaria.

\section{Caracterização das empresas}

Foram estabelecidas quatro variáveis para caracterizar as empresas: setor de atividade econômica (indústria, serviços ou comércio), porte (micro, pequena, média ou grande) (19, 20), modalidade de adesão ao PAT (autogestão, gestão terceirizada do tipo refeição transportada ou gestão terceirizada do tipo preparo e distribuição de refeição na empresa) e supervisão de nutricionista (sim ou não).

O porte foi definido com base no número de funcionários: foram consideradas como micro as empresas com até 19 funcionários no caso da indústria e

TABELA 1. Recomendações nutricionais do Guia Alimentar para a População Brasileira, 2005

\begin{tabular}{lc}
\hline Nutriente & $\begin{array}{c}\text { Recomendação } \\
\left(\% \text { do valor calórico total) }{ }^{a}\right.\end{array}$ \\
\hline Carboidrato & 55 a 75 \\
Proteína & 10 a 15 \\
Gorduras totais & 15 a 30 \\
Gordura saturada & $<10$ \\
Gordura poliinsaturada & 6 a 10 \\
Gordura trans & $<1$ \\
Colesterol & $300 \mathrm{mg} /$ dia \\
Açúcares livres & $<10$ \\
Frutas e hortaliças & $400 \mathrm{~g} / \mathrm{dia}$ \\
\hline Fonte: Ministério da Saúde. & \\
a Valor calórico total diário $=2$ o 000 calorias. A recomendação quanto à ingestão de colesterol e \\
frutas e hortaliças é expressa em mg/dia e g/dia, respectivamente.
\end{tabular}

até nove funcionários no caso dos outros setores; como pequenas as empresas com 20 a 99 funcionários na indústria e 10 a 49 funcionários nos outros setores; como médias as empresas com 100 a 499 funcionários na indústria e 50 a 499 nos outros setores; e como grandes as empresas com 500 funcionários ou mais para todos os setores.

\section{Análise estatística}

Para caracterizar as refeições e as empresas, foram calculadas a freqüência absoluta e a relativa e medidas de tendência central (médias) e de dispersão (desvios padrão e valores mínimos e máximos). Inicialmente, foi realizada uma análise de agrupamento (cluster) segundo porte, setor de atividade, modalidade de adesão ao PAT e presença de nutricionista, pois essas variáveis estão altamente associadas, possuindo características semelhantes. Esse tipo de análise permite agrupar objetos similares para o conjunto de variáveis definidas (21).

As diferenças entre os agrupamentos de empresas quanto às médias das variáveis alimentares foram calculadas com o teste $\mathrm{t}$ de Student. $\mathrm{O}$ teste do qui-quadrado $\left(\chi^{2}\right)$ foi utilizado para avaliar a relação entre o tipo de agrupamento e a adequação dos cardápios. Utilizou-se o programa estatístico SPSS (versão 10.0) para a elaboração do banco de dados e a análise de dados. O ponto de corte adotado para significância estatística foi $P<0,05$.

O estudo foi aprovado pelo comitê de ética da Faculdade de Saúde Pública da Universidade de São Paulo (USP) e foi realizado em conformidade com a resolução 196/96 do Conselho Nacional de Saúde.

\section{RESULTADOS}

Foram avaliadas 72 empresas, sendo $41,7 \%$ do setor do comércio, $51,4 \%$ de médio e grande porte e $61,1 \%$ cadastradas na modalidade de autogestão da unidade de alimentação e nutrição. A supervisão de nutricionista esteve presente em $61,1 \%$ das empresas. As empresas foram distribuídas em dois agrupamentos (tabela 2): o primeiro incluiu principalmente empresas de micro e pequeno porte, sem supervisão de nutricionista, do setor de comércio, tendo autogestão como modalidade do programa. O segundo foi composto principalmente por empresas de médio e grande porte, com supervisão de nutricionista, do setor industrial e de serviços, com gestão terceirizada.

A análise do valor calórico das refeições, independentemente do agrupamento, mostrou valor médio de 1 195,03 \pm 350,87 kcal/refeição, dividido em $50,95 \pm 8,69 \%$ de carboidratos, $18,85 \pm 4,38 \%$ de proteínas e 30,18 \pm $8,31 \%$ de gorduras totais. Em relação 
TABELA 2. Características gerais das empresas cadastradas no Programa de Alimentação do Trabalhador incluídas no estudo sobre oferta alimentar no trabalho, São Paulo (SP), Brasil, 2004

\begin{tabular}{|c|c|c|c|c|c|c|}
\hline \multirow[b]{2}{*}{ Característica } & \multicolumn{2}{|c|}{ Empresas $(n=72)$} & \multicolumn{2}{|c|}{ Agrupamento $1(n=40)^{\mathrm{a}}$} & \multicolumn{2}{|c|}{ Agrupamento $2(n=32)^{\mathrm{b}}$} \\
\hline & No. & $\%$ & No. & $\%$ & No. & $\%$ \\
\hline \multicolumn{7}{|l|}{ Setor } \\
\hline Indústria & 21 & 29,2 & 2 & 5,0 & 19 & 54,4 \\
\hline Serviços & 21 & 29,2 & 9 & 22,5 & 12 & 37,5 \\
\hline Comércio & 30 & 41,7 & 29 & 72,5 & 1 & 3,1 \\
\hline \multicolumn{7}{|l|}{ Porte ${ }^{c}$} \\
\hline Micro & 8 & 11,1 & 8 & 20,0 & 0 & 0 \\
\hline Pequena & 27 & 37,5 & 24 & 60,0 & 3 & 9,4 \\
\hline Média & 28 & 38,9 & 8 & 20,0 & 20 & 62,5 \\
\hline Grande & 9 & 12,5 & 0 & 0 & 9 & 28,1 \\
\hline \multicolumn{7}{|l|}{ Modalidade de adesão ao PAT } \\
\hline Autogestão & 44 & 61,1 & 38 & 95,0 & 6 & 18,8 \\
\hline Gestão terceirizada: refeição transportada & 4 & 5,6 & 2 & 5 & 2 & 6,3 \\
\hline Gestão terceirizada: preparo e distribuição de refeição & 24 & 33,3 & 0 & 0 & 24 & 75,0 \\
\hline \multicolumn{7}{|l|}{ Supervisão de nutricionista } \\
\hline Sim & 44 & 61,1 & 15 & 37,5 & 29 & 90,6 \\
\hline Não & 28 & 38,9 & 25 & 62,5 & 3 & 9,4 \\
\hline
\end{tabular}

Inclui principalmente empresas de micro e pequeno porte do setor de comércio, tendo autogestão como modalidade do programa e sem supervisão de nutricionista.

b Inclui principalmente empresas de médio e grande porte do setor industrial e de serviço, com gestão terceirizada e supervisão de nutricionista.

c Microempresa: até 19 funcionários na indústria e até nove nos outros setores; pequena: de 20 a 99 funcionários na indústria e de 10 a 49 nos outros setores; média: de 100 a 499 funcionários na indústria e de 50 a 499 nos outros setores; grande: acima de 500 funcionários em todos os setores.

à oferta de gordura nos cardápios, a média de gordura poliinsaturada foi de $3,87 \pm 1,91 \%$ do valor calórico total (VCT); de gordura saturada, 9,09 \pm $4,12 \%$ do VCT; de gordura trans, $1,71 \pm 1,34 \%$ do VCT; e de colesterol, $156,03 \pm 74,02 \mathrm{mg}$. A média da participação dos açúcares livres no VCT das refeições foi de $10,52 \pm 7,38 \%$. O valor médio de frutas e hortaliças ofertadas foi de 142,81 $\pm 103,5 \mathrm{~g}$.

Grande parte dos cardápios avaliados teve baixa oferta de gordura poliinsaturada $(83,3 \%)$ e de frutas e hortaliças $(63,9 \%)$ em relação às recomendações do Guia. Por outro lado, observou-se que muitos cardápios ofereceram excesso de gorduras totais $(47,2 \%)$, gordura saturada $(31,9 \%)$, gorduras trans $(51,4 \%)$, colesterol $(62,5 \%)$ e açúcares livres $(47,2 \%)$.

Os resultados relativos às características do valor nutricional das refeições oferecidas segundo análise de agrupamento das empresas são apresentados na tabela 3. As empresas do agrupamento 2 ofereceram, em média, refeições com maior valor energético
$(P<0,001)$, maior porcentagem de gorduras poliinsaturadas $(P<0,001)$, maior quantidade de colesterol $(P=$ $0,015)$ e maior quantidade de frutas e hortaliças $(P<0,001)$ que as empresas do agrupamento 1 .

Na tabela 4 são apresentados os resultados referentes à adequação dos cardápios oferecidos em relação às metas de ingestão de nutrientes sugeridas pelo Guia Alimentar para a População Brasileira. As empresas do agrupamento 2 ofereceram refeições mais adequadas em relação à oferta de

TABELA 3. Valor nutricional médio das grandes refeições oferecidas segundo agrupamento das empresas, São Paulo (SP), Brasil, 2004

\begin{tabular}{|c|c|c|c|c|c|}
\hline \multirow[b]{2}{*}{ Nutriente/alimento } & \multicolumn{2}{|c|}{ Agrupamento $1^{\mathrm{a}}$} & \multicolumn{2}{|c|}{ Agrupamento $2^{b}$} & \multirow[b]{2}{*}{$P^{c}$} \\
\hline & Média & Desvio padrão & Média & Desvio padrão & \\
\hline Energia (kcal) & 1035,96 & 336,65 & 1393,86 & 256,77 & $<0,001$ \\
\hline Carboidratos (\%) & 50,47 & 7,96 & 51,56 & 9,62 & 0,599 \\
\hline Proteínas (\%) & 19,41 & 4,97 & 18,15 & 3,47 & 0,226 \\
\hline Lipídios totais (\%) & 30,11 & 8,42 & 30,27 & 8,31 & 0,932 \\
\hline Lipídios poliinsaturados (\%) & 3,27 & 1,90 & 4,61 & 1,68 & 0,003 \\
\hline Ácidos graxos trans (\%) & 1,13 & 1,03 & 1,44 & 0,88 & 0,187 \\
\hline Colesterol (mg) & 137,26 & 74,99 & 179,61 & 66,68 & 0,015 \\
\hline Açúcares livres (\%) & 9,92 & 8,29 & 11,27 & 6,09 & 0,444 \\
\hline Frutas e hortaliças (g) & 89,91 & 82,9 & 208,93 & 88,13 & $<0,001$ \\
\hline
\end{tabular}

a $n=40$. Inclui principalmente empresas de micro e pequeno porte do setor de comércio, tendo autogestão como modalidade do programa e sem supervisão de nutricionista.

b $n=32$. Inclui principalmente empresas de médio e grande porte do setor industrial e de serviço, com gestão terceirizada e supervisão de nutricionista.

${ }^{c}$ Nível descritivo do teste de $t$ de Student $(P<0,05)$. 
TABELA 4. Adequação dos cardápios de grande refeição das empresas em relação às recomendações do Guia Alimentar para a População Brasileiraa , São Paulo (SP), Brasil, 2004

\begin{tabular}{|c|c|c|c|c|c|}
\hline \multirow[b]{2}{*}{ Nutrientes/alimentos } & \multicolumn{2}{|c|}{$\begin{array}{l}\text { Agrupamento } 1 \\
\quad(n=40)^{\mathrm{b}}\end{array}$} & \multicolumn{2}{|c|}{$\begin{array}{l}\text { Agrupamento } 2 \\
(n=32)^{c}\end{array}$} & \multirow[b]{2}{*}{$P^{d}$} \\
\hline & Adequada & $\%$ & Adequada & $\%$ & \\
\hline Lipídios poliinsaturados (6 a $10 \%$ do $\mathrm{VCT}^{\mathrm{e}}$ ) & 5 & 12,5 & 7 & 21,9 & 0,289 \\
\hline Lipídios saturados $\left(<10 \%\right.$ do $\left.\mathrm{VCT}^{\mathrm{e}}\right)$ & 25 & 62,5 & 24 & 75,0 & 0,258 \\
\hline Ácidos graxos trans $\left(<1 \%\right.$ do $\left.\mathrm{VCT}^{\mathrm{e}}\right)$ & 23 & 57,5 & 12 & 37,5 & 0,092 \\
\hline Colesterol $(<120 \mathrm{mg})$ & 19 & 47,5 & 8 & 25,0 & 0,050 \\
\hline Açúcares livres (<10\% do $\mathrm{VCT}^{\mathrm{e}}$ ) & 24 & 60,0 & 14 & 43,8 & 0,170 \\
\hline Frutas e hortaliças (> $160 \mathrm{~g}$ ) & 5 & 12,5 & 21 & 65,6 & $<0,001$ \\
\hline
\end{tabular}

a Brasil, Ministério da Saúde (8).

b Empresas de micro e pequeno porte, sem supervisão de nutricionista, do setor de comércio, tendo autogestão como modalidade do programa.

c Empresas de médio e grande porte, com supervisão de nutricionista, do setor industrial e de serviço, com gestão terceirizada.

d Nível descritivo do teste do $\chi^{2}(P<0,05)$.

e Valor calórico total.

frutas e hortaliças do que as empresas do agrupamento $1(P<0,001)$. Em contrapartida, as empresas do agrupamento 1 apresentaram maior adequação da quantidade de colesterol quando comparadas às do agrupamento $2(P=0,05)$.

\section{DISCUSSÃO}

Os resultados obtidos neste estudo mostram a ocorrência de elevados percentuais de inadequação para todas as variáveis estudadas em relação às metas de ingestão de nutrientes recomendadas pela OMS para a prevenção de doenças crônicas e a promoção de alimentação saudável. Quando analisadas as características nutricionais dos cardápios, verificou-se que as empresas de ambos os agrupamentos apresentaram oferta média de energia superior à recomendação diária de energia para uma grande refeição, de 30 a $40 \%$ de 2000 kcal. Em relação à distribuição percentual dos macronutrientes, observou-se, nos dois agrupamentos, uma oferta de carboidratos inferior à recomendada, uma oferta de proteínas superior à recomendada $\mathrm{e}$ uma oferta de gorduras totais próxima do limite superior da recomendação. Outros trabalhos que avaliaram a oferta de alimentos de uma grande refeição também encontraram cardápios com conteúdo de energia e proteína superior ao recomendado e redução no percentual calórico proveniente dos carboidratos $(12,22)$. Esses resultados condizem com a mudança de padrão alimentar no Brasil, evidenciando o maior consumo de carnes, leite e derivados ricos em gorduras e a queda no consumo de cereais (23).

Observou-se que as empresas do primeiro agrupamento ofereceram, em média, um pouco mais da metade da recomendação da OMS de frutas e hortaliças. Nos últimos anos, vários trabalhos vêm evidenciando a importância desses alimentos para a prevenção de doenças crônicas não transmissíveis $(24,25)$. Apesar disso, alguns estudos têm mostrado o baixo consumo de frutas e hortaliças pela população brasileira (25) e a baixa oferta desses alimentos em restaurantes no ambiente de trabalho (12).

Em relação ao perfil lipídico das refeições, observou-se baixa oferta de gorduras poliinsaturadas pelas empresas de ambos os agrupamentos. Em média, a oferta de gordura saturada esteve dentro das recomendações, porém ainda há um percentual elevado de empresas que oferece excesso desse nutriente.

Observou-se uma oferta elevada de colesterol e gordura trans nos cardápios oferecidos pelas empresas de ambos os agrupamentos, em especial do segundo. Apesar de os dados disponíveis em relação ao teor de gordura trans nas tabelas brasileiras de composição de alimentos ainda serem limita- dos, o que pode ter levado à subestimação da quantidade oferecida deste nutriente nas refeições, foi possível identificar excesso na sua oferta.

O teor dos açúcares livres nas refeições oferecidas também foi elevado, em especial no agrupamento 2, que apresentou uma oferta, em média, maior do que a recomendada. Esse resultado pode ser explicado pela baixa oferta de frutas como sobremesa e pela elevada oferta de doces, sucos adoçados e refrigerantes. Outros estudos também encontraram elevada oferta e consumo de açúcar pelos trabalhadores no local de trabalho, principalmente de refrigerantes, bebidas adoçadas e de açúcar como ingrediente de preparações (22, 26). Os dados obtidos na Pesquisa de Orçamento Familiar realizada no Brasil em 2002 e 2003 (27) mostraram que o açúcar e o refrigerante possuem elevada participação, 11,9 e 1,5\%, respectivamente, no total de calorias disponíveis para consumo pelas famílias. Os dados sugerem que as unidades de alimentação e nutrição oferecem refeições que acompanham a tendência de consumo alimentar e de comportamento da população brasileira em geral.

É importante ressaltar que, na análise dos cardápios, foi considerada a oferta para os trabalhadores dos diferentes alimentos que os compunham, não levando em conta desperdícios e sobras, o que é uma limitação do estudo. Além disso, deve ser considerada a possibilidade de ter havido 
algum viés de aferição, pois algumas empresas não possuíam registro per capita dos alimentos ou preparações.

As refeições oferecidas pelas empresas de pequeno e médio porte, do setor de comércio, sem supervisão de nutricionista, apresentaram os piores resultados em relação às quantidades de gordura saturada e poliinsaturada e de frutas e hortaliças. Estes achados indicam a necessidade de uma ação direcionada a empresas inscritas no PAT que apresentam esse perfil. Iniciativas recentes, de diferentes setores do governo brasileiro, têm buscado adequar os parâmetros nutricionais do PAT ao atual perfil epidemiológico e nutricional dos trabalhadores brasileiros. O Guia Alimentar para a População Brasileira (8) traz as primeiras diretrizes alimentares oficiais, que endossam as recomendações nutricionais da OMS. Além disso, a Portaria Interministerial $\mathrm{n}^{\circ} 66$, de 25 de agosto de 2006, que alterou os parâmetros nutricionais do PAT, reforçou a necessi- dade das empresas inscritas no PAT de oferecer uma refeição com padrão alimentar adequado às necessidades biológicas e sociais dos indivíduos, ao determinar parâmetros nutricionais para alguns nutrientes que são reconhecidamente relacionados com o desenvolvimento de doenças crônicas não transmissíveis (18).

Os cardápios oferecidos pelas empresas cadastradas no PAT apresentaram várias barreiras para a efetiva prevenção de doenças crônicas não transmissíveis por meio da oferta de alimentação saudável. Colares (28) ressalta a necessidade de um maior envolvimento de entidades da área de alimentação coletiva, como conselhos e associações de nutricionistas e empresas fornecedoras e prestadoras de serviços de alimentação coletiva, entre outros, com o objetivo de melhorar a fiscalização do PAT e fazer com que o programa efetivamente contribua para a melhoria das condições de saúde do trabalhador.
O Guia Alimentar para a População Brasileira (8) traz, entre suas diretrizes, a recomendação de que sejam incorporados aos programas públicos de alimentação e nutrição os princípios de alimentação saudável, o que caracteriza um desafio diante dos resultados encontrados. Considerando que o local de trabalho é um contexto social onde o comportamento do trabalhador em relação à saúde pode ser estimulado e mantido (7), é imprescindível que os gestores das empresas e os responsáveis técnicos pelas unidades de alimentação e nutrição cadastradas no PAT sejam sensibilizados com relação à oportunidade que os locais de trabalho oferecem para promover a saúde dos trabalhadores.

Agradecimentos. Este estudo foi financiado pelo Conselho Nacional de Desenvolvimento Científico e Tecnológico (CNPq) através do processo 471136/03-4.

\section{REFERÊNCIAS}

1. Monteiro CA, Moura EC, Jaime PC, Lucca A, Florindo AA, Figueiredo ICR, et al. Monitoramento de fatores de risco para doenças crônicas por entrevistas telefônicas. Rev Saude Publica. 2005;39(1):47-57.

2. Santos CRB, Portella ES, Avila SS, Soares EA Fatores dietéticos na prevenção e tratamento de comorbidades associadas à síndrome metabólica. Rev Nutr. 2006;19(3):389-401.

3. Millen BE, Quatromoni PA, Pencina M, Kimokoti R, Nam BH, Cobain S, et al. Unique dietary patterns and chronic disease risk profiles of adult men: the Framingham nutrition studies. J Am Diet Assoc. 2005;105(11):1723-34

4. World Health Organization (WHO). Diet, nutrition and the prevention of chronic diseases. Report of a joint WHO/FAO Expert Consultation. Genebra: WHO; 2003. (Technical Report Series, $n^{\circ}$ 916)

5. Waxman A; World Health Assembly. WHO global strategy on diet, physical activity and health. Food Nutr Bull. 2004;25(3):292-302.

6. Matos MFD, Silva NAS, Pimenta AJM, Cunha AJLA. Prevalência dos fatores de risco para doença cardiovascular em funcionários do centro de pesquisas da Petrobras. Arq Bras Cardiol. 2004;82(1):1-8.

7. Karlehagen S, Ohlson CG. Primary prevention of cardiovascular disease by an occupational health service. Prev Med. 2003;37(3): 219-25.
8. Brasil, Ministério da Saúde. Guia alimentar para a população brasileira: promovendo a alimentação saudável. Brasília: Ministério da Saúde; 2006.

9. Brasil, Ministério do Trabalho e Emprego. Programa de Alimentação do Trabalhador (PAT) [site da Internet]. Brasília; Ministério do Trabalho e Emprego; 2005. Disponível em: http://www.mte.gov.br/Empregador/pat/ default.asp. Acessado em 7 de janeiro de 2005.

10. Brasil, Ministério do Trabalho e Emprego. Portaria da Secretaria de Inspeção do Trabalho $\mathrm{n}^{\circ} 03$ de 1 de março de 2002. Artigo $1^{\circ}$ : Do objetivo do programa de alimentação do trabalhador (PAT). Brasília: Ministério do Trabalho e Emprego [site da Internet]. Disponível em: http:/ / www.mte.gov.br/Empregador/pat/ Legislacao/Default.asp. Acessado em 4 de maio de 2006.

11. Freire RBM, Salgado RS. Avaliação de cardápios oferecidos a trabalhadores horistas. Mundo Saude. 1998;22(5):298-301.

12. Savio KEO, Costa THM, Miazaki E, Schmitz BAS. Avaliação do almoço servido a participantes do programa de alimentação do trabalhador. Rev Saude Publica. 2005;39(2): 148-55.

13. Veloso IS, Santana VS. Impacto nutricional do programa de alimentação do trabalhador no Brasil. Rev Panam Salud Publica. 2002;11(1): 24-31.
14. Instituto Brasileiro de Geografia e Estatística (IBGE). Estimativa da população dos municípios em 2006 [site da Internet]. Disponível em: http://www.ibge.gov.br/home/estatistica/ populacao/contagem $2007 /$ popmunic 2007 layoutTCU14112007.pdf. Acessado em 01 de outubro de 2006.

15. Instituto Brasileiro de Geografia e Estatística (IBGE). Estudo nacional de despesas familiares-ENDEF, 1974-1975. Rio de Janeiro: IBGE; 1978.

16. São Paulo, Universidade de Campinas. Tabela de composição de alimentos [site da Internet]. Disponível em: http://www.unicamp.br/ nepa/taco/. Acessado em 4 de maio 2006.

17. United States Department of Agriculture. Nutrient Data Laboratory [site da internet]. Disponível em: http://www.nal.usda.gov/fnic/ foodcomp $/$. Acessado em $1^{\circ}$ de fevereiro de 2005.

18. Brasil, Ministério do Trabalho e Emprego. Portaria Interministerial $n^{\circ} 66$, de 25 de agosto de 2006. Altera os parâmetros nutricionais do Programa de Alimentação do Trabalhador (PAT). Brasília: Ministério do Trabalho e Emprego; 2006. Disponível em: http://www. mte.gov.br/legislacao/portarias/2006/p 20061205_193.pdf. Acessado em dezembro de 2007.

19. Moura JB. Avaliação do Programa de Alimentação do Trabalhador no estado de Pernam- 
buco, Brasil. Rev Saude Publica. 1986;20(2): 115-28.

20. Serviço brasileiro de apoio às micro e pequenas empresas (SEBRAE). Legislação básica da micro e pequena empresa [site da Internet]. Disponível em: http://www.sebrae.com.br/ $\mathrm{br} /$ aprendasebrae/estudosepesquisas.asp. Acessado em 17 de março de 2006.

21. Pereira JCR. Análise de dados qualitativos. $3^{a}$ ed. São Paulo: EDUSP; 2004.

22. Amorim MMA, Junqueira RG, Jokl L. Adequação nutricional do almoço self-service de uma empresa de Santa Luzia, MG. Rev Nutr. 2005;18(1):145-56.

23. Mondini L, Monteiro CA. Mudanças no padrão de alimentação. Em: Monteiro CA, org. Velhos e novos males da saúde no Brasil: a evolução do país e de suas doenças. São
Paulo: Hucitec/Núcleo de Pesquisas Epidemiológicas em Nutrição e Saúde, Universidade de São Paulo; 1995. Pp. 79-89.

24. Williams MT, Hord NG. The role of dietary factors in cancer prevention: beyond fruits and vegetables. Nutr Clin Pract. 2005;20(4): 451-9.

25. Jaime PC, Monteiro CA. Fruit and vegetable intake by Brazilian adults, 2003. Cad Saude Publica. 2005;21(Suppl 1):519-24.

26. Gambardella AMD. O programa de alimentação do trabalhador frente às recomendações nutricionais para esse segmento específico da população: área metropolitana de São Paulo [dissertação]. São Paulo: Universidade de São Paulo; 1990.

27. Levy-Costa RB, Sichieri R, Pontes N dos S, Monteiro CA. Disponibilidade domiciliar de alimentos no Brasil: distribuição e evolução (1974-2003). Rev Saude Publica. 2005;39(4): 530-40.

28. Colares LGT. Evolução e perspectivas do programa de alimentação do trabalhador no contexto político brasileiro. Nutrire Rev Soc Bras Aliment Nutr. 2005;29:141-58.

Manuscrito recebido em 6 de maio de 2006. Aceito em versão revisada em 30 de janeiro de 2007.

ABSTRACT Objective. To compare the nutritional value of meals provided by companies participating in the Workers' Meal Program in the city of São Paulo, Brazil, to the nutritional recommendations and guidelines established by the Ministry of Health for the

Nutritional information of meals supplied by companies participating in the Workers' Meal Program in São Paulo, Brazil

Methods. The 72 companies studied were grouped according to economic sector (industrial, services, or commerce), size (micro, small, medium, or large), meal preparation modality (prepared on-site by the company itself, on-site by a hired caterer, or off-site by a hired caterer), and supervision by a dietitian (yes or no). The per capita amount of food was determined based on the lunch, dinner, and supper menus for three days. The nutritional value of the meals was defined by the amount of calories, carbohydrates, protein, total fat, polyunsaturated fat, saturated fat, trans fat, sugars, cholesterol, and fruits and vegetables.

Results. Most of the menus were deficient in the number of fruits and vegetables $(63.9 \%)$ and amount of polyunsaturated fat $(83.3 \%)$, but high in total fat $(47.2 \%)$ and cholesterol (62.5\%). Group 2, composed of mostly medium and large companies, supervised by a dietician, belonging to the industrial and/or service sectors, and using a hired caterer, on averaged served meals with higher calorie content $(P<0.001)$, higher percentage of polyunsaturated fat $(P<0.001)$, more cholesterol $(P=0.015)$, and more fruits and vegetables $(P<0.001)$ than Group 1 , which was composed of micro and small companies from the commercial sector, that prepare the meals themselves on-site, and are not supervised by a dietitian. Regarding the nutrition guidelines set for the Brazilian population, Group 2 meals were better in terms of fruit and vegetable servings $(P<0.001)$. Group 1 meals were better in terms of cholesterol content $(P=0.05)$.

Conclusions. More specific action is required targeting company officers and managers in charge of food and nutrition services, especially in companies without dietitian supervision.

Key words Nutrition programs and policies; health promotion; chronic disease; menu planning, workplace, Brazil. 